\title{
Efecto del uso de las redes sociales sobre las actividades académicas en estudiantes universitarios
}

\section{Dr. Cirilo Duarte Félix ${ }^{1}$, Dr. María Guadalupe Almada Quintero² ${ }^{2}$ Lic. Armando Medina Wahnnatah ${ }^{3}$}

\begin{abstract}
Resumen
El presente análisis propicia la reflexión en torno al uso de las redes sociales en la vida universitaria, es indudable que la incursión de las redes sociales en la sociedad, está produciendo de forma acelerada nuevas formas de comunicación e interacción entre las personas. Lo cual desencadena consecuencias para los usuarios. El objetivo de este trabajo es analizar el uso que hacen los estudiantes universitarios de las redes sociales y conocer los resultados de su utilización y los comportamientos socialmente inadecuados. Se analizan aspectos relacionados con las consecuencias que puede producir el uso de las redes sociales. Entre las principales conclusiones a las que se puede llegar con este estudio destacamos que las redes sociales y el uso de internet puede producir algunos problemas en los usuarios sobre todo cuando su uso va dirigido mayormente al entretenimiento. Resulta relevante retomar la discusión del uso de redes adecuadas para el provecho académico.
\end{abstract}

Palabras Clave: Adicción, redes sociales, Actividades académicas, dependencia, aplicaciones móviles, celular.

\footnotetext{
${ }^{1}$ Doctor en Investigación Educativa en la Instituto del Desierto de Santa Ana. Campus Navojoa. Docente en la Universidad Estatal de Sonora. duarte606@hotmail.com

${ }^{2}$ Doctorado en Planeación y Liderazgo Educativo por la Universidad del Valle de México. Campus Hermosillo. Docente en la Universidad Estatal de Sonora. magaq@hotmail.com

${ }^{3}$ Licenciado en Derecho por la Universidad de Sonora. Docente en la Universidad Estatal de Sonora. Campus Navojoa. licarmandomedinaw@hotmail.com
} 


\title{
http://revistainvestigacionacademicasinfrontera.com
}

\begin{abstract}
The present analysis encourages reflection on the use of social networks in university life, there is no doubt that the incursion of social networks in society is rapidly producing new forms of communication and interaction between people. Which triggers consequences for users. The objective of this work is to analyze the use that university students make of social networks and to know the results of their use and socially inappropriate behaviors. We analyze aspects related to the consequences that the use of social networks can produce. Among the main conclusions that can be reached with this study, we emphasize that social networks and the use of the Internet can produce some problems for users, especially when their use is mainly aimed at entertainment. It is relevant to resume the discussion of the use of appropriate networks for academic benefit.
\end{abstract}

\section{Keywords}

Addiction, social networks, academic activities, dependency, mobile applications, cellphone.

\section{Introducción}

Los avances tecnológicos siguen creciendo en gran medida para facilitar nuestras vidas, tanto que la sociedad se ha vuelto más dependiente del uso de la tecnología. El uso excesivo de las nuevas aplicaciones como los celulares, está ocasionando, que los jóvenes no respeten espacios como la escuela, que a menudo se ve cómo interrumpen al maestro que está explicando una clase, con 


\section{http://revistainvestigacionacademicasinfrontera.com}

señales acústicas o risas por ver una publicación en Facebook ${ }^{4}$, recibir un mensaje de Whatsapp ${ }^{5}$, una foto de Instagram ${ }^{6}$ o responder un Twitter ${ }^{7}$.

Hoy uno de los problemas que más presencia está teniendo en torno al uso de celulares en los jóvenes universitarios, es la adicción a las redes sociales y video juegos. Generando situaciones problemáticas como: falta de atención en clase, reducción de productividad escolar, potenciar conductas individualistas, degradación de lenguaje, adicción a ellas. (Caldevilla, 2010).

Las etiquetas "adicción a Internet”, "trastorno de adicción a Internet”, "uso patológico de Internet”, "trastorno de dependencia de la red", "ciberadicción”, etc. se han utilizado para describir el mismo concepto: un uso inadecuado de Internet que produce descuido de otras áreas de la vida (Widyanto y Griffiths, 2006), dificultades para concentrarse y síndrome de abstinencia.

\subsection{Adicción}

La Organización Mundial de la Salud (OMS) define una adicción como una enfermedad física y psicoemocional que crea una dependencia o necesidad hacia una sustancia, actividad o relación. Se caracteriza por un conjunto de signos y síntomas, en los que se involucran factores biológicos, genéticos, psicológicos y sociales. Es una enfermedad progresiva y fatal, caracterizada por episodios continuos de descontrol, distorsiones del pensamiento y negación ante la enfermedad.

Autores como Echeburúa y Corral (2009), sostienen que cualquier conducta placentera es susceptible de convertirse en un comportamiento adictivo. De este modo, la adicción puede originarse debido a la intervención de una sustancia, pero también puede motivarse por la interacción de una conducta que conlleva la pérdida de control por parte del sujeto, el

\footnotetext{
${ }^{4}$ Es una plataforma de red social mediante la cual los usuarios pueden mantenerse en contacto continuo con un grupo de amistades e intercambiar información.

${ }^{5}$ Es el nombre de una aplicación que permite enviar y recibir mensajes instantáneos a través de un teléfono móvil (celular).

${ }^{6}$ Es una aplicación que actúa como red social ya que permite a sus usuarios subir cualquier tipo de fotos y videos.

${ }^{7}$ Es una red de microblogging que permite escribir y leer mensajes en Internet que no superen los 140 caracteres.
} 
Núm. 28

ISSN: 2007-8870

\section{http://revistainvestigacionacademicasinfrontera.com}

establecimiento de una relación de dependencia, una tolerancia hacia esa conducta y la interferencia grave de ésta en la vida cotidiana

\section{Niveles de adicción}

Existen diferentes niveles de adicción ${ }^{8}$ :

\section{No consumo:}

Nunca ha utilizado las redes en particular.

\section{Consumo experimental:}

Ha utilizado las redes sociales una o algunas veces.

El motivo es la curiosidad.

\section{Consumo esporádico:}

Consumo infrecuente e irregular, generalmente en ocasiones especiales o cuando se presenta la oportunidad.

\section{Consumo habitual:}

La utilización sigue un patrón predecible, que puede ser frecuente o infrecuente.

El consumidor busca la utilización de las redes sociales o bien participar en las actividades de su grupo de amigos.

Generalmente tienen sensación de controlar el consumo. ("Yo cuando quiera lo dejo").

\section{Dependencia:}

La utilización es regular y generalmente frecuente.

El consumidor experimenta dependencia por utilizar las redes sociales.

Siente que su consumo está fuera de su control.

\footnotetext{
${ }^{8}$ Se contextualizaron los niveles de adicción a niveles de adicción a las redes sociales
} 


\section{http://revistainvestigacionacademicasinfrontera.com}

El consumo continúa a pesar de las consecuencias negativas.

\subsubsection{Adicción al teléfono.}

No existe una categoría expresa en el Manual Diagnóstico y Estadístico de las Enfermedades Mentales (DSM-V ${ }^{9}$ ) que catalogue el excesivo uso del móvil como un trastorno de la adicción, pero existen características con las que se comparten grandes similitudes con otros trastornos como el de dependencia de sustancias y encontramos el síndrome de abstinencia, la tolerancia y la pérdida de control por no usarlo.

Investigaciones realizadas por Forbes ${ }^{10}$, revelan que México es uno de los países con mayores problemas en el uso de dispositivos móviles en los salones de clase, indicando que el crecimiento anual de uso de Smartphone es por encima de $40 \%$ anual, con 40 millones de teléfonos utilizados por jóvenes entre 15 y 29 años.

Otro dato estadístico encontrado es que en promedio, una persona ve su celular unas 150 veces al día (suena exagerado pero esta cifra representa unas 10 veces por hora), algunas muchas más. Las cifras de Forbes indican que casi el $30 \%$ de los estudiantes lo usa en horas de clases

Investigaciones de Igarashi, Motoyoshi y Yoshida (2007), estudiaron los componentes de la auto atribución de adicción al móvil, encontrando a tres: percepción de uso excesivo, reacciones emocionales (por ejemplo al no recibir inmediatamente respuesta de mensajes de texto) y motivación para el mantenimiento de las relaciones.

\subsubsection{Adicción al Internet o ciberespacio}

La adicción a internet o ciberadicción se puede definir según Héctor Basile (2006) como "la pérdida del control frente al uso racional de Internet". Podemos saber si alguien es ciberadicto cuando, de forma excesiva, hace uso de internet y no piensa en nada más que en eso.

\footnotetext{
${ }^{9}$ Manual diagnóstico y estadístico de trastornos mentales, quinta edición, septiembre 2016

${ }^{10}$ revista especializada en el mundo de los negocios y las finanzas. Fundada en 1917 por B. C. Forbes.
} 


\section{http://revistainvestigacionacademicasinfrontera.com}

En México, la adicción al internet se ha incrementado considerablemente ya que ocho de cada diez mexicanos de seis años o más que utilizan celular tiene un teléfono inteligente, según datos de la Encuesta Nacional sobre Disponibilidad y Uso de Tecnologías de la Información en los Hogares del INEGI ${ }^{11}$; incrementando de 60.6 millones de usuarios a 64.7 millones del 2016 al 2017, lo que representa más de la mitad de la población.

Según declaraciones de Gabriel Richaud, director de IAB ${ }^{12}$ México (Interactive Advertising Bureau), considera que el incremento de los mexicanos al uso del internet se debe a que el acceso a la adquisición de los dispositivos (teléfonos inteligentes) y la gran cantidad de puntos de conexión, ha hecho que los usuarios estén permanentemente conectados.

\section{Red Social}

En sentido amplio, "una red social es una estructura social formada por personas o entidades conectadas y unidas entre sí por algún tipo de relación o interés común. El término se atribuye a los antropólogos británicos Alfred Radcliffe-Brown y Jhon Barnes” (Ponce, 2012) .

Actualmente, las redes sociales son consideradas la principal actividad de Internet en México, utilizado por el 83\% de internautas mexicanos. (Asociación de Internet MX, 2017).

Una de las principales causas por la que los estudiantes no relacionan el empleo de redes sociales con el ámbito académico, es debido fundamentalmente a que las instituciones educativas no otorgan a éstas el papel fundamental que poseen en el día a día de los alumnos.

En la investigación de Consumo de Medios Digitales en México se indica que $70 \%$ de los usuarios en México están registrados al menos en una red social, siendo los jóvenes entre 19 y 25 años quienes más se comunican a través de estos recursos (IAB, 2013), siendo esta la edad promedio de los estudiantes universitarios en México.

\footnotetext{
${ }^{11}$ Instituto Nacional de Estadística y Geografía.

${ }^{12}$ Es el principal organismo a nivel global que representa a la industria de la publicidad digital y marketing interactivo
} 


\section{http://revistainvestigacionacademicasinfrontera.com}

En este contexto, surgen las siguientes interrogantes: ¿Para qué utilizan las redes sociales digitales los jóvenes universitarios? ¿Qué tipo de herramientas tecnológicas utilizan? ¿Son importantes para realizar actividades escolares?, entre otras. En México, se han realizado estudios que muestran que actualmente es común que los estudiantes ingresen a las universidades con algún aparato tecnológico: celular, computadora portátil, tableta, entre otros (Crovi, Garay, López y Portillo, 2013); Existen escasas investigaciones referentes al uso que le dan a las redes sociales, por lo que se considera relevante que los estudiantes expresen su punto de vista, experiencias y prácticas en relación al uso que le dan en el aula de clase.

\section{Conclusiones}

Sin duda el análisis enfocado a la utilización de las redes sociales en la educación en épocas actuales resulta de suma importancia, su uso es cada vez más frecuente por los estudiantes por lo que su implementación por parte de los docentes se vuelve imprescindible como una herramienta de trabajo y de apoyo a las estrategias didácticas y a la propia interacción en el medio en el cual se desarrollan los jóvenes.

En este mismo sentido, del análisis practicado se puede señalar, que las nuevas generaciones de jóvenes integran mayormente las tecnologías digitales en su quehacer cotidiano de manera natural lo cual no necesariamente conlleva a que sepan utilizarlas y que las apliquen en su formación como una herramienta de gran provecho. Se puede apreciar en la mayoría de los casos que este uso de las redes sociales por los estudiantes mayormente va encaminado a las actividades recreativas y de socialización, los estudiantes destinan diariamente una gran cantidad de tiempo a esta práctica, lo cual se puede considerar como una gran área de oportunidad, es importante promover la reflexión en el ámbito educativo hacia el uso de las redes sociales como herramienta académica, como un recurso educativo, que contribuya a la formación destacando la inmediatez de la información, la 


\section{http://revistainvestigacionacademicasinfrontera.com}

posibilidad de la comunicación constante así como el proceso de acompañamiento durante el proceso formativo.

Las redes sociales pueden ser un complemento para el desarrollo de los entornos virtuales de aprendizaje aplicado, claro está en una forma consciente, reflexiva y profesional de parte de los estudiantes, comprendiendo que su uso e implementación debe ser en el marco de la mejora continua en contribución a su profesionalización, sin dejar de lado la implementación de programas de incremento de las habilidades sociales con el fin de mejorar la interacción social en el contexto universitario.

\section{Referencias}

Basile, H. (2006). Adicción a Internet: Ciberadicción. Alcmeon, Revista Argentina de Clínica Neuropsiquiátrica, 74-90.

Widyanto, L., \& Griffiths, M. (2006). Internet Addiction: A Critical Review. International Journal of Mental Health and Addiction, 31-51.

Caldevilla Domínguez, D. (2010). Las Redes Sociales. Documentación de las Ciencias de la Información, 45-68.

Domínguez, F., \& López, R. (2015). Uso de las redes sociales digitales entre los jóvenes universitarios en México. Hacia la construcción de un estado del conocimiento (20042014). Revista de Comunicación , 48-69.

Echeburúa, E., \& de Corral, P. (2009). Adicción a las nuevas tecnologías y a las redes sociales en jóvenes: un nuevo reto. ADICCIONES, 91-96.

Jasso Medrano, J. L., López Rosales, F., \& Díaz Loving, R. (2017). Conducta adictiva a las redes sociales y su relación con el uso problemático del móvil. Psicológica - Psychological Research Records, 2832-2838. 


\section{http://revistainvestigacionacademicasinfrontera.com}

Luengo López, A. (2004). Adicción a Internet: conceptualización y propuesta de intervención. Revista Profesional Española de Terapia Cognitivo-Conductual (ASETECCS), 22-52.

Marín Díaz, V., \& Sampedro Requena, B. (2015). ¿Son adictos a las redes sociales los estudiantes universitarios? . Revista Complutense de Educación , 233-251.

Ponce, I. (17 de Abril de 2012). Ministerio de Educación, Cultura y Deporte. Obtenido de http://recursostic.educacion.es//observatorio/web/ca/internet/web-20//1043-redessociales? start $=1$

Sánchez Rodríguez, J., Ruiz Palmero, J., \& Sánchez Rivas, E. (2015). Uso problemático de las redes sociales en estudiantes universitarios. Revista Complutense de Educación, 159-174.

\section{Como citar este articulo}

Cirilo Duarte Félix- María Guadalupe Almada Quintero-Armando Medina Wahnnatah. Efecto del uso de las redes sociales sobre las actividades académicas en estudiantes universitarios. RIASF. Núm. 28, Julio-diciembre (2018), ISSN 2007-8870. pp. xx-xx. 